\title{
Low Cardiac Output Secondary to a Malpositioned Umbilical Venous Catheter: Value of Targeted Neonatal Echocardiography
}

\author{
Dany E. Weisz, MD ${ }^{1}$ Wei Bing Poon, MD ${ }^{1}$ Andrew James, MD ${ }^{1}$ Patrick J. McNamara, MD ${ }^{1,2,3}$ \\ ${ }^{1}$ Department of Paediatrics, Hospital for Sick Children, Toronto, \\ Ontario, Canada \\ 2 Physiology and Experimental Medicine Program, Hospital for Sick \\ Children, Toronto, Ontario, Canada \\ Address for correspondence Patrick J. McNamara, MD, Department of \\ Neonatology, The Hospital for Sick Children, University of Toronto, 555 \\ University Avenue, Toronto, ON M5G1X8, Canada \\ (e-mail: patrick.mcnamara@sickkids.ca).
}

${ }^{3}$ Department of Physiology, University of Toronto, Toronto,

Ontario, Canada

Am J Perinatol Rep 2014;4:23-28.

\begin{abstract}
Keywords

- targeted neonatal echocardiography

- umbilical venous catheter

- low cardiac output

- atrial septal defect

Systemic hypotension is common in very low birthweight preterm infants but the nature of the precipitating cause may be unclear. Targeted neonatal echocardiography (TnEcho) is being increasingly used to support hemodynamic decisions in the neonatal intensive care unit (NICU), including identifying impairments in the transitional circulation of preterm infants, providing timely re-evaluation after institution of therapies and evaluating the placement of indwelling catheters. We present a case of a preterm infant with systemic hypotension and low cardiac output secondary to a large transatrial shunt induced by a malpositioned umbilical venous catheter. Repositioning of the line led to resolution of the hemodynamic disturbance and clinical instability, highlighting the utility of TnEcho in the NICU.
\end{abstract}

The clinical approach to the evaluation and management of hemodynamic instability in very low birthweight (VLBW) infants lacks scientific validation and often fails to consider physiologic factors. Systemic hypotension is common but methods used to determine the underlying etiology and accurately evaluate end-organ perfusion are limited. ${ }^{1}$ Targeted neonatal echocardiography (TnEcho) is being increasingly used to support hemodynamic decisions in VLBW infants as part of a framework of physiology-based care., ${ }^{2,3}$ We present a case which highlights the utility of TnEcho in determining the nature of cardiovascular compromise and monitoring treatment response.

\section{Case}

The index case was born at 26 weeks 5 days gestation with a birthweight of $1,020 \mathrm{~g}$. His mother was a healthy 29 -year-old woman, gravida 3 and para 1, who presented to the commu- nity hospital with spontaneous preterm labor and progressed to an unassisted vaginal delivery. The baby was intubated and mechanically ventilated at 2 minutes of life and exogenous surfactant was administered. Cord blood gas results were normal. Ampicillin and gentamicin were started for empiric therapy of possible sepsis. He was transported to the tertiary hospital neonatal intensive care unit (NICU).

He was hemodynamically stable on arrival and was ventilated using a volume-targeted pressure-limited mode set to a tidal volume of $4 \mathrm{~mL} / \mathrm{kg}$, positive end-expiratory pressure $5 \mathrm{~cm} \mathrm{H}_{2} \mathrm{O}$, rate 40 , and $\mathrm{FiO}_{2} 0.21$. He required a peak inspiratory pressure of 14 to $17 \mathrm{~cm} \mathrm{H}_{2} \mathrm{O}$. Umbilical arterial and venous (UVC) catheters were inserted. The UVC was secured at $8 \mathrm{~cm}$. Chest and abdominal radiographs were performed to evaluate line placement ( - Fig. 1). The tip of the UVC was identified $1 \mathrm{~cm}$ above the level of the diaphragm on the lateral chest radiograph. The UVC was withdrawn $1 \mathrm{~cm}$ and resecured at $7 \mathrm{~cm}$. At 5 hours of life, noninvasive and invasive received

January 27, 2013 accepted after revision

March 5, 2013

published online

March 3, 2014
DOI http://dx.doi.org/ 10.1055/s-0034-1368090. ISSN 2157-6998.
Copyright $\odot 2014$ by Thieme Medical Publishers, Inc., 333 Seventh Avenue, New York, NY 10001, USA. Tel: +1(212) 584-4662.
License terms

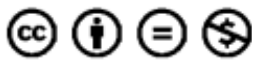




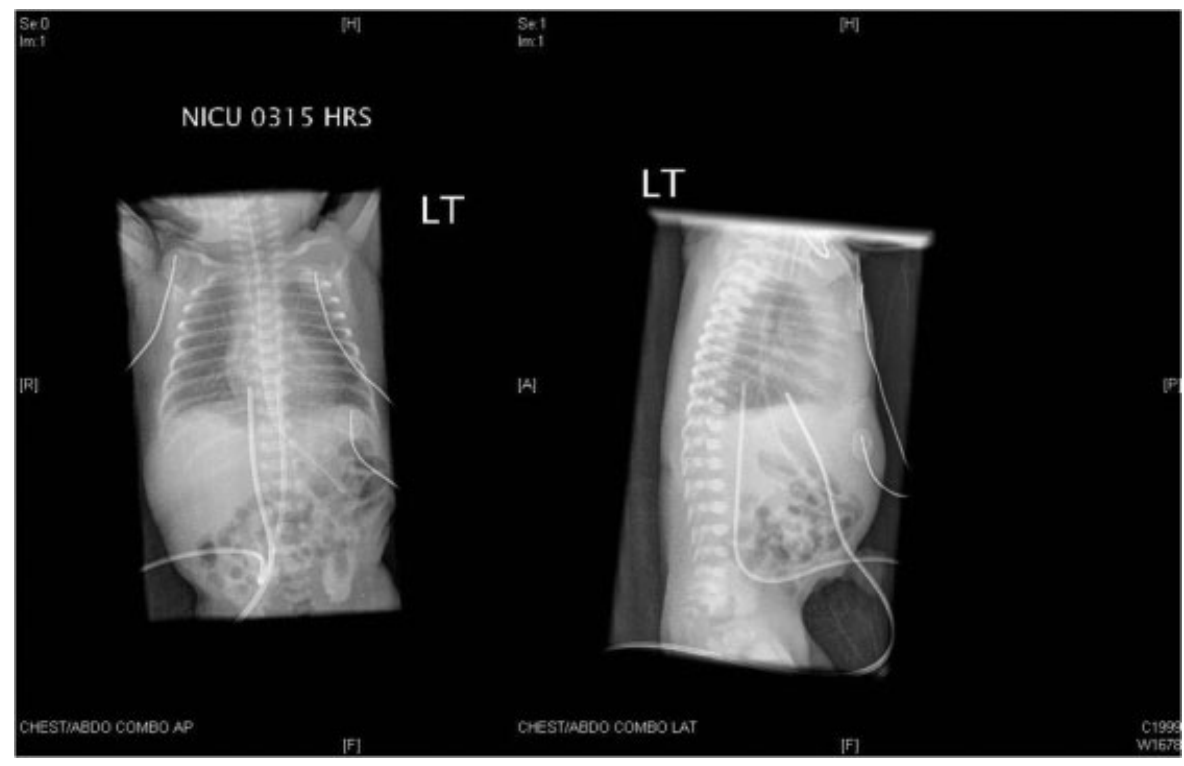

Fig. 1 Anterior-posterior chest radiograph of the infant after UVC and UAC placement demonstrating the tip of the UVC just above the ninth thoracic vertebrae and the tip of the UAC positioned at the eighth thoracic vertebrae. The lateral chest radiograph demonstrates the tip of the UVC $1 \mathrm{~cm}$ above the diaphragm. The UVC was therefore pulled out $1 \mathrm{~cm}$ and resecured. UAC, umbilical arterial catheter; UVC, umbilical venous catheter.

arterial pressures $(\mathrm{mm} \mathrm{Hg}$ ) were both noted to be low at $31 / 17$ (22) and 35/18 (25), respectively. Pre- and postductal oxygen saturations were $93 \%$ on minimal ventilator support and $\mathrm{FIO}_{2}$ 0.21 . Clinical examination revealed a heart rate (HR) of 161 beats per minute, low amplitude central and peripheral pulses, and a quiet precordium with no heart murmur. Arterial blood gas (ABG) revealed a mild metabolic acidosis (pH 7.20, $\mathrm{PaCO}_{2}$ 53, $\mathrm{HCO}_{3}{ }^{-}$20, base deficit 8). Two $10 \mathrm{~mL} / \mathrm{kg}$ boluses of normal saline and a dobutamine infusion were administered. The infant's clinical status further deteriorated with an invasive arterial pressure of 29/12 (19), HR 161, diminished central pulses, absent peripheral pulses, and onset of oliguria. A repeat $A B G$ revealed worsening metabolic acidosis ( $\mathrm{pH} 7.22, \mathrm{PaCO}_{2} 43, \mathrm{HCO}_{3}{ }^{-} 17$, base deficit 10 ).

At 16 hours of life, a TnEcho consultation was requested to assess for a hemodynamically significant patent ductus arteriosus (PDA) in view of refractory hypotension. Left ventricle (LV) output was found to be low (-Fig. 2) at $140 \mathrm{~mL} / \mathrm{kg} / \mathrm{min}$ (normal $170-350 \mathrm{~mL} / \mathrm{kg} / \mathrm{min}$ ). LV fractional shortening was $46 \%$ (normal 25-55\%). The ductus arteriosus was $2.6 \mathrm{~mm}$ with bidirectional pulsatile flow, although $80 \%$ was left to right. There was absent diastolic flow in the celiac artery and middle cerebral artery (MCA) (- Table 1). The UVC was noted to be intracardiac, traversing the patent foramen ovale such
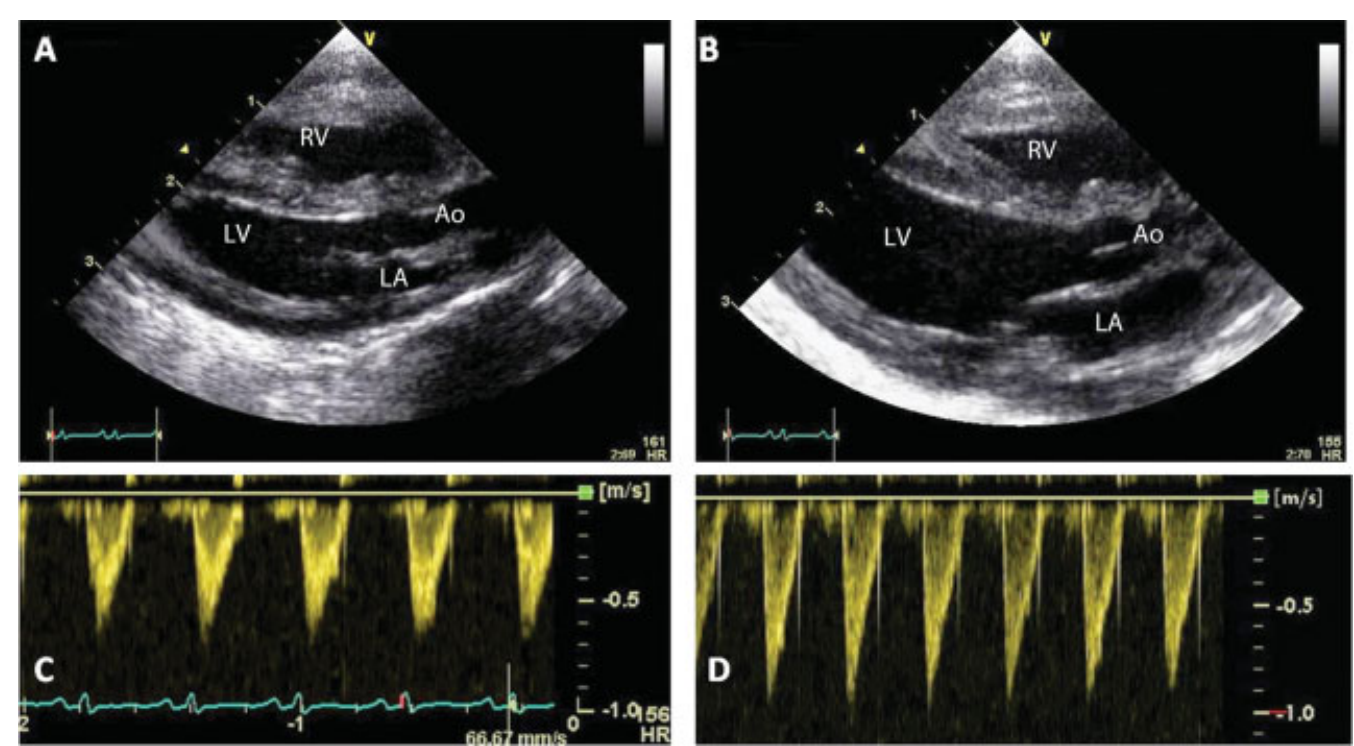

Fig. 2 Two-dimensional long-axis view of left atrium and ventricle showing low chamber size before (A) compared with after (B) manipulation of the umbilical venous catheter. Pulse-wave Doppler interrogation of left ventricular outflow tract from apical five-chamber view demonstrating low velocity time integral (flow) before (C) compared with after (D) catheter manipulation. Ao, aorta; LA, left atrium; LV, left ventricle; RV, right ventricle. 
that the tip was in the left atrium, creating a large $4.2 \mathrm{~mm}$ atrial septal defect (ASD) with exclusive left-to-right transatrial flow at a maximum velocity of $0.7 \mathrm{~cm} / \mathrm{s}$ (- Fig. 3). Under real-time echocardiographic guidance, the UVC was withdrawn until the catheter tip was identified at the right atrial (RA)/inferior vena cava (IVC) junction. At this point, the UVC was resecured, at a length of $6 \mathrm{~cm}$. Within 30 minutes of repositioning of the UVC, the arterial pressure normalized. A repeat TnEcho was performed 1 hour later which demonstrated improved left ventricular output (LVO) to $270 \mathrm{~mL} / \mathrm{kg} / \mathrm{min}$ and a reduction in the ASD size to $1.3 \mathrm{~mm}$ (-Figs. 2 and 3). Celiac artery and MCA diastolic flow had normalized. Over the subsequent 8 hours, the urine output and metabolic acidosis improved and the dobutamine was successfully discontinued.

\section{Discussion}

This is the first reported case of cardiorespiratory instability in a VLBW infant attributable to a high order of magnitude transatrial shunt secondary to a malpositioned UVC. The clinical presumption was that the etiology of the hypotension was hypovolemia or myocardial dysfunction; however, there was no clinical improvement with therapeutic strategies to improve preload (with normal saline) and myocardial contractility (with dobutamine).

Bedside echocardiography evaluation performed by a trained neonatologist revealed that the patient had low LVO secondary to a large left-to-right transatrial shunt. The atrial defect was artificially enlarged by the malpositioned UVC which traversed and stretched open the foramen ovale leading to a large conduit. The biologic consequences of a large left-to-right transatrial shunt include decreased left ventricular preload and low LVO, both of which were evident on the echocardiography study. Although it is not possible to quantify the volume of the transatrial shunt, the temporal relationship between repositioning of the UVC, reduction in the size of the transatrial shunt, increase in left ventricular cavity dimension and LVO, and finally improvement in clinical indices of systemic perfusion and blood pressure support this biophysiological association. This is further strengthened by

Table 1 Targeted neonatal echocardiogram evaluation before and after manipulation of umbilical venous catheter

\begin{tabular}{|c|c|c|c|c|}
\hline Component & Parameter & Initial TnEcho & Follow-up TnEcho & Reference range $^{\mathrm{a}}$ \\
\hline \multirow[t]{2}{*}{ LV systolic function } & LVFS, \% & 46 & 42 & $25-55$ \\
\hline & LV output, $\mathrm{mL} / \mathrm{kg} / \mathrm{min}$ & 140 & 270 & $170-350$ \\
\hline \multirow[t]{4}{*}{ LV diastolic function } & IVRT, ms & 42 & 40 & $35-55$ \\
\hline & E:A ratio & 0.81 & 0.85 & $<1.0$ (preterm infant) \\
\hline & Mitral regurgitation & Absent & Absent & Absent \\
\hline & LA:Ao & 1.15 & 1.6 & $<1.5: 1$ \\
\hline \multirow[t]{3}{*}{ RV function } & RVET:PAAT & 4 & 3.8 & $<4$ \\
\hline & $\begin{array}{l}\text { Septal wall motion } \\
\text { during diastole }\end{array}$ & Flattening & Normal & Normal \\
\hline & RVSp (mm Hg) & 34 & 18 & \\
\hline \multirow[t]{3}{*}{ Atrial level shunt } & ASD size, $\mathrm{mm}$ & 4.2 & 1.3 & $<3.0$ \\
\hline & Flow (direction) & Left to right & Left to right & \\
\hline & Flow (max. velocity), $\mathrm{cm} / \mathrm{s}$ & 0.7 & 1.6 & \\
\hline \multirow[t]{3}{*}{ PDA } & Transductal diameter & $\begin{array}{l}2.6 \mathrm{~mm}(2 \mathrm{D}) ; 2.8 \mathrm{~mm} \\
\text { (color Doppler) }\end{array}$ & $\begin{array}{l}2.6 \mathrm{~mm}(2 \mathrm{D}) \text {; } \\
2.8 \mathrm{~mm} \\
\text { (color Doppler) }\end{array}$ & \\
\hline & Flow (direction) & $\begin{array}{l}\text { Bidirectional } \\
\text { (80\% left to right) }\end{array}$ & Left to right & \\
\hline & Flow (pattern) & Pulsatile (unrestrictive) & Pulsatile (unrestrictive) & \\
\hline \multirow[t]{3}{*}{ Systemic blood flow } & Superior mesenteric artery & Reversed & Antegrade & Antegrade \\
\hline & Celiac artery & Absent & Antegrade & Antegrade \\
\hline & Middle cerebral artery & Absent & Antegrade & Antegrade \\
\hline UVC tip position & & $\begin{array}{l}\text { Deep in left atrium, } \\
\text { traversing the ASD }\end{array}$ & IVC-RA junction & IVC-RA junction \\
\hline
\end{tabular}

Abbreviations: ASD, atrial septal defect; E:A, early to late (atrial) filling ratio; IVC, inferior vena cava; IVRT, isovolumetric relaxation time; LA:Ao, left atrium to aorta ratio; LV, left ventricle; LVFS, left ventricular fractional shortening; PDA, patent ductus arteriosus; PAAT:RVET, pulmonary artery acceleration time to right ventricular ejection time ratio; PDA, patent ductus arteriosus; RA, right atrial; RV, right ventricle; RVSp, right ventricular systolic pressure; 2D, two-dimensional; TnEcho, targeted neonatal echocardiography; UVC, umbilical venous catheter.

alf applicable. 

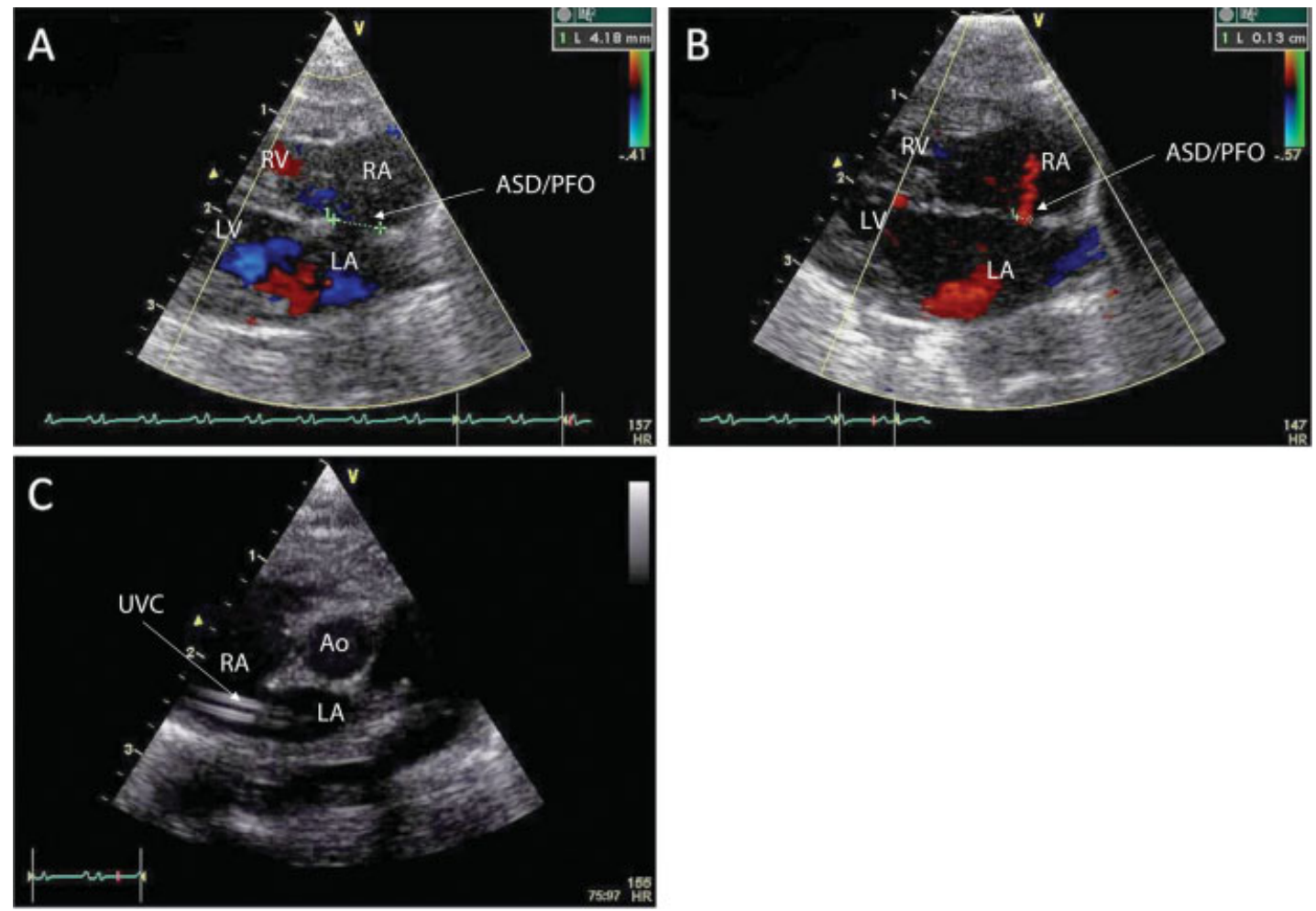

Fig. 3 Two-dimensional and color Doppler subcostal view of atrial septum showing large atrial septal defect before (A) compared with after (B) manipulation of the umbilical venous catheter. The catheter is seen crossing the atrial septum into the left atrium on a short-axis view (C). Ao, aorta; ASD/PFO, atrial septal defect/patent foramen ovale; LA, left atrium; LV, left ventricle; RA, right atrium; RV, right ventricle; UVC, umbilical venous catheter.

studies demonstrating that ASDs may have a significant hemodynamic impact and associated clinical morbidity in preterm infants. Evans and Iyer found a significant correlation between atrial shunt diameter and ventricular outputs in VLBW preterm infants. ${ }^{4}$ Shunting was minimal when the ASD diameter was less than $2 \mathrm{~mm}$ and gradually increased, such that when the ASD exceeded $4 \mathrm{~mm}$ (as in our patient), the average pulmonary-to-systemic flow ratio (Qp:Qs) was 1.5:1. Other studies have confirmed that the size of the atrial defect in VLBW infants shortly after birth is positively correlated with increasing right ventricular output and is associated with reduced left ventricular output., ${ }^{5,6}$ In addition, the presence of the UVC traversing the foramen ovale and entering the left atrium may have impacted the interatrial shunting to a degree that is not conceptualized by the size of the ASD alone. Potential mechanisms include catheter-related mitral outflow obstruction or the Coanda effect of the catheter enhancing the atrial shunt. ${ }^{7,8}$ In our case, the TnEcho revealed the true physiologic nature of the deterioration, allowed realtime guidance to reposition the catheter, and permitted timely re-evaluation of the hemodynamic improvement.

TnEcho is increasingly being used to support clinical hemodynamic decisions in the NICU. ${ }^{2}$ Systemic hypotension occurs in one-third of preterm infants but determining the underlying etiology may be difficult. ${ }^{1}$ The changes in cardiovascular physiology in the immediate transitional period are highly complex and influenced by right and left ventricular afterload, postnatal myocardial adaptation, ductal and atrial shunts, and the effects of mechanical ventilation and surfactant. TnEcho may provide additional information regarding the physiologic or anatomical nature of cardiovascular impairment in preterm infants, which may either support the clinical suspicion or reveal novel insights. ${ }^{3}$ An increasing body of evidence supports its use in the management of a PDA ${ }^{9,10}$ and in infants with pulmonary hypertension. ${ }^{11}$ This evidence should guide a more physiologically relevant approach to cardiovascular decision making and the role of TnEcho in supporting these decisions needs to be explored further.

This report also demonstrates that TnEcho is valuable in identifying aberrant placement of indwelling catheters, corroborating the results of previous studies. ${ }^{12}$ The ideal location of the UVC tip is at the IVC-RA junction. Verification of UVC tip position by conventional radiography is often inaccurate. ${ }^{13}$ With echocardiography, the IVC-RA junction can be easily visualized using a parasagittal subcostal view, enabling realtime, accurate line placement and reducing the need for radiographs. ${ }^{2,14}$ The adverse effects of malpositioned umbilical venous catheters have been well described. ${ }^{15}$ However, the potential adverse hemodynamic effect of atrial shunting due to a UVC malpositioned in the left atrium has not previously been reported. TnEcho offers a safe, accurate, timely, and readily accessible alternative to radiological evaluation.

Although the role of TnEcho appears promising, the risks of its implementation, including misdiagnosis, necessitate standards for training, and clinical practice. Recently, a 
writing group of expert pediatric cardiologists and neonatologists was convened to develop guidelines for training and clinical practice. The duration of the TnEcho training lasts, at minimum, 4 to 6 months within a shared collaborative neonatology and cardiology program. ${ }^{16}$ Standards and guidelines for clinical practice should be agreed upon by both pediatric cardiologists and neonatologists at practicing institutions.

In summary, we have demonstrated that a malpositioned catheter traversing the atrial septum may cause cardiorespiratory instability. TnEcho is a potentially useful tool in the NICU to identify malpositioned catheters, characterize the nature of hemodynamic instability, and provide timely reevaluation after intervention.

\section{Conflict of Interest}

None

\section{References}

1 Al-Aweel I, Pursley DM, Rubin LP, Shah B, Weisberger S, Richardson DK. Variations in prevalence of hypotension, hypertension, and vasopressor use in NICUs. J Perinatol 2001;21(5):272-278

2 El-Khuffash AF, McNamara PJ. Neonatologist-performed functional echocardiography in the neonatal intensive care unit. Semin Fetal Neonatal Med 2011;16(1):50-60

3 Sehgal A. Haemodynamically unstable preterm infant: an unresolved management conundrum. Eur J Pediatr 2011;170(10): 1237-1245

4 Evans N, Iyer P. Incompetence of the foramen ovale in preterm infants supported by mechanical ventilation. J Pediatr 1994; 125(5 Pt 1):786-792

5 Evans N, Kluckow M. Early determinants of right and left ventricular output in ventilated preterm infants. Arch Dis Child Fetal Neonatal Ed 1996;74(2):F88-F94
6 Evans N, Iyer P. Assessment of ductus arteriosus shunt in preterm infants supported by mechanical ventilation: effect of interatrial shunting. J Pediatr 1994;125(5 Pt 1):778-785

7 Ginghină C. The Coandă effect in cardiology. J Cardiovasc Med (Hagerstown) 2007;8(6):411-413

8 Chao K, Moises VA, Shandas R, Elkadi T, Sahn DJ, Weintraub R. Influence of the Coanda effect on color Doppler jet area and color encoding. In vitro studies using color Doppler flow mapping. Circulation 1992;85(1):333-341

9 Jain A, Sahni M, El-Khuffash A, Khadawardi E, Sehgal A, McNamara PJ. Use of targeted neonatal echocardiography to prevent postoperative cardiorespiratory instability after patent ductus arteriosus ligation. J Pediatr 2012;160(4):584-589

10 Carmo KB, Evans N, Paradisis M. Duration of indomethacin treatment of the preterm patent ductus arteriosus as directed by echocardiography. J Pediatr 2009;155(6):819-822

11 Subhedar NV, Hamdan AH, Ryan SW, Shaw NJ. Pulmonary artery pressure: early predictor of chronic lung disease in preterm infants. Arch Dis Child Fetal Neonatal Ed 1998;78(1):F20-F24

12 Ades A, Sable C, Cummings S, Cross R, Markle B, Martin G. Echocardiographic evaluation of umbilical venous catheter placement. J Perinatol 2003;23(1):24-28

13 Raval NC, Gonzalez E, Bhat AM, Pearlman SA, Stefano JL. Umbilical venous catheters: evaluation of radiographs to determine position and associated complications of malpositioned umbilical venous catheters. Am J Perinatol 1995;12(3):201-204

14 Jain A, McNamara PJ, Ng E, El-Khuffash A. The use of targeted neonatal echocardiography to confirm placement of peripherally inserted central catheters in neonates. Am J Perinatol 2012;29(2): 101-106

15 Ramasethu J. Complications of vascular catheters in the neonatal intensive care unit. Clin Perinatol 2008;35(1):199-222

16 Mertens L, Seri I, Marek J, et al; Writing Group of the American Society of Echocardiography; European Association of Echocardiography; Association for European Pediatric Cardiologists. Targeted Neonatal Echocardiography in the Neonatal Intensive Care Unit: practice guidelines and recommendations for training. Writing Group of the American Society of Echocardiography (ASE) in collaboration with the European Association of Echocardiography (EAE) and the Association for European Pediatric Cardiologists (AEPC). J Am Soc Echocardiogr 2011;24(10):1057-1078 